\title{
Dependence of the Photocurrent of a Schottky-Barrier Solar Cell on the Back Surface Recombination Velocity and Suggestion for a Structure with Improved Performance
}

\author{
Avigyan Chatterjee, Ashim Kumar Biswas, and Amitabha Sinha \\ Department of Physics, University of Kalyani, Kalyani, West Bengal 741235, India \\ Correspondence should be addressed to Amitabha Sinha; asinha333@gmail.com
}

Received 25 June 2015; Revised 20 November 2015; Accepted 30 November 2015

Academic Editor: Sundaram Senthilarasu

Copyright (C) 2015 Avigyan Chatterjee et al. This is an open access article distributed under the Creative Commons Attribution License, which permits unrestricted use, distribution, and reproduction in any medium, provided the original work is properly cited.

\begin{abstract}
Though Schottky-barrier solar cells have been studied extensively previously, not much work has been done recently on these cells, because of the fact that conventional $p-n$ junction silicon solar cells have much higher efficiency and have attracted the attention of most of the researchers. However, the Schottky-barrier solar cells have the advantage of simple and economical fabrication process. In this paper, the effect of back surface recombination velocity on the minority carrier distribution and the spectral response of a Schottky-barrier silicon solar cell have been investigated and, based on this study, a new design of the cell with a back surface field has been suggested, which is expected to give much improved performance.
\end{abstract}

\section{Introduction}

After the development of the first silicon p-n junction solar cell [1], tremendous amount of research and development work have been done to improve its efficiency. Blakers and Green [2] reported a 20\% efficiency silicon solar cell and later Wang et al. [3] developed a $24 \%$ efficiency silicon solar cell. Many new designs of silicon solar cells were developed over the years. Schottky-barrier silicon solar cell is one such device, which has been investigated by various researchers. Pulfrey and McOuat calculated the maximum theoretical conversion efficiency of Schottky-barrier solar cells [4] and concluded that greater simplicity of fabrication of these devices would make them attractive as solar energy converters. A general model for the analysis of Schottkybarrier solar cells, taking into account the optical properties, carrier recombination effects, semiconductor minority carrier properties, and effect of series resistance, was presented by McOuat and Pulfrey [5]. Though Schottky-barrier solar cells have much less efficiency than conventional p-n junction solar cells, their advantage lies in the simple and economical fabrication process. In the present work, the effect of back surface recombination velocity on the minority carrier distribution and the spectral response of a Schottky-barrier solar cell (SBSC) have been studied and a new design for the SBSC structure has been suggested. This new structure, which takes its analogy from the back surface field $\mathrm{n}^{+} \mathrm{pp}^{+}$ silicon solar cells, yields much improved performance than the conventional SBSC.

\section{Analysis}

A detailed analytical study of a Schottky-barrier silicon solar cell (SBSC) has been undertaken earlier by the authors [6], the salient features of which are discussed here in brief. The diagram of a metal (n-type) silicon Schottky-barrier solar cell is shown in Figure 1, in which light is incident on the front metal surface and an ohmic contact exists at the back. The light photons are absorbed in the semiconductor, giving rise to electron hole pairs, which are separated by the built-in field that exists at the junction. This is responsible for the photocurrent in the device.

To obtain analytical expressions for the minority carrier concentration and the photocurrent of the solar cell, 


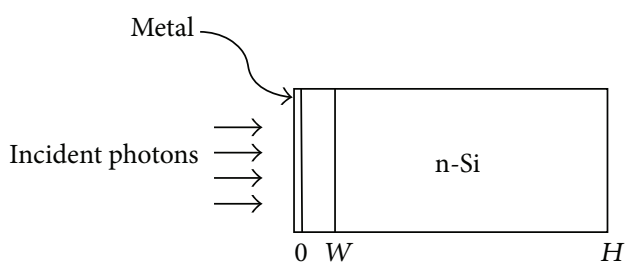

FIGURE 1: Schematic diagram of a SBSC.

the continuity equation and the current density equation are combined to obtain the following differential equation $[6,7]$ :

$$
D_{p} \frac{d^{2}\left(p_{n}-p_{n 0}\right)}{d x^{2}}+\alpha F T \exp (-\alpha x)-\frac{\left(p_{n}-p_{n 0}\right)}{\tau_{p}}=0,
$$

where $F$ is the flux of incident photons, $T$ is the transmission coefficient, $\alpha$ is the absorption coefficient of silicon, $D_{p}$ is the diffusion constant for holes, $\tau_{p}$ is the lifetime of holes, $p_{n}$ is the hole concentration in $n$ region, and $p_{n 0}$ is the thermal equilibrium concentration of holes in this region.
The general solution for this differential equation is

$$
\begin{aligned}
\left(p_{n}-p_{n 0}\right)= & A \cosh \left(\frac{x}{L_{p}}\right)+B \sinh \left(\frac{x}{L_{p}}\right) \\
& -\frac{\alpha F T \tau_{p}}{\left(\alpha^{2} L_{p}^{2}-1\right)} \exp (-\alpha x),
\end{aligned}
$$

where $L_{p}=\sqrt{D_{p} \tau_{p}}$ is the diffusion length for holes. $A$ and $B$ are constants, which can be evaluated using the boundary conditions [6]:

$$
\begin{aligned}
\left(p_{n}-p_{n 0}\right) & =0 \quad \text { at }[x=W], \\
S\left(p_{n}-p_{n 0}\right) & =-D_{p} \frac{d\left(p_{n}-p_{n 0}\right)}{d x} \quad[x=H],
\end{aligned}
$$

where $S$ is the back surface recombination velocity. Substituting the values of the constants, an expression for the excess minority carrier holes may be written as [6]

$$
\begin{aligned}
& \left(p_{n}-p_{n 0}\right)=\left[\frac{\alpha F T \tau_{p}}{\left(\alpha^{2} L_{p}^{2}-1\right)}\right][\exp (-\alpha W)]\left[\cosh \left(\frac{x-W}{L_{p}}\right)-\exp (-\alpha(x-W))\right. \\
& \left.-\frac{\left(S L_{p} / D_{p}\right)\left[\cosh \left(H^{\prime} / L_{p}\right)-\exp \left(-\alpha H^{\prime}\right)\right]+\sinh \left(H^{\prime} / L_{p}\right)+\alpha L_{p} \exp \left(-\alpha H^{\prime}\right)}{\left(S L_{p} / D_{p}\right) \sinh \left(H^{\prime} / L_{p}\right)+\cosh \left(H^{\prime} / L_{p}\right)} \sinh \left(\frac{x-W}{L_{p}}\right)\right] .
\end{aligned}
$$

The corresponding expression for the hole current density is obtained as [7]

$$
\begin{aligned}
J_{p}= & {\left[\frac{q F T \alpha L_{p}}{\left(\alpha^{2} L_{p}^{2}-1\right)}\right][\exp (-\alpha W)] } \\
& \cdot\left[\alpha L_{p}-\frac{\left(S L_{p} / D_{p}\right)\left[\cosh \left(H^{\prime} / L_{p}\right)-\exp \left(-\alpha H^{\prime}\right)\right]+\sinh \left(H^{\prime} / L_{p}\right)+\alpha L_{p} \exp \left(-\alpha H^{\prime}\right)}{\left(S L_{p} / D_{p}\right) \sinh \left(H^{\prime} / L_{p}\right)+\cosh \left(H^{\prime} / L_{p}\right)}\right],
\end{aligned}
$$

where $q$ is the charge of electron and $S$ is the back surface recombination velocity. $W$ is the edge of the depletion region in the semiconductor. Here, $H^{\prime}=H-W$.

The photocurrent contribution from the depletion region of the cell is then given by [7]

$$
J_{\mathrm{dr}}=q T F[1-\exp (-\alpha W)] .
$$

The spectral response of the Schottky-barrier solar cell is then given by [7]

$$
\operatorname{SR}(\lambda)=\frac{J_{\mathrm{dr}}(\lambda)+J_{p}(\lambda)}{q F(\lambda) T(\lambda)} .
$$

It is observed from (5) and (6) that the excess minority carrier hole concentration and the photocurrent are dependent on the back surface recombination velocity $S$.

\section{Results and Discussions}

Based on the mathematical expressions presented above, calculations were performed to obtain the different graphs. The values of different parameters used here are $N_{D}=$ $10^{15} \mathrm{~cm}^{-3}, F=3.5 \times 10^{15} \mathrm{~cm}^{-2}, T=0.5$, and the thickness of the cell $H=300 \mu \mathrm{m}$. The mobility and the lifetime of minority carrier holes are doping dependent. The values of doping dependent lifetime have been obtained from 


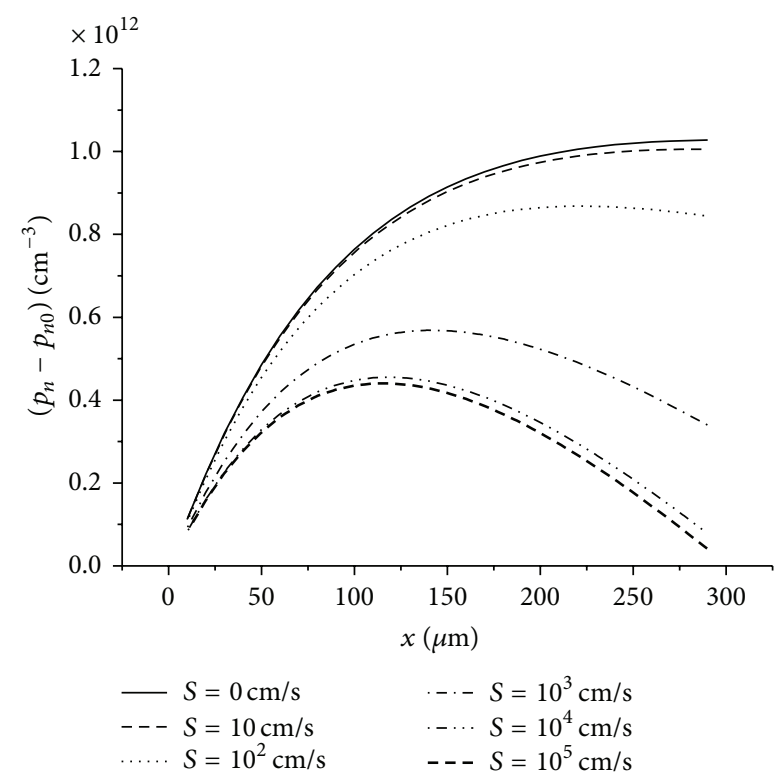

FIGURE 2: Excess minority carrier distribution in semiconductor layer, as a function of the back surface recombination velocity $S$.

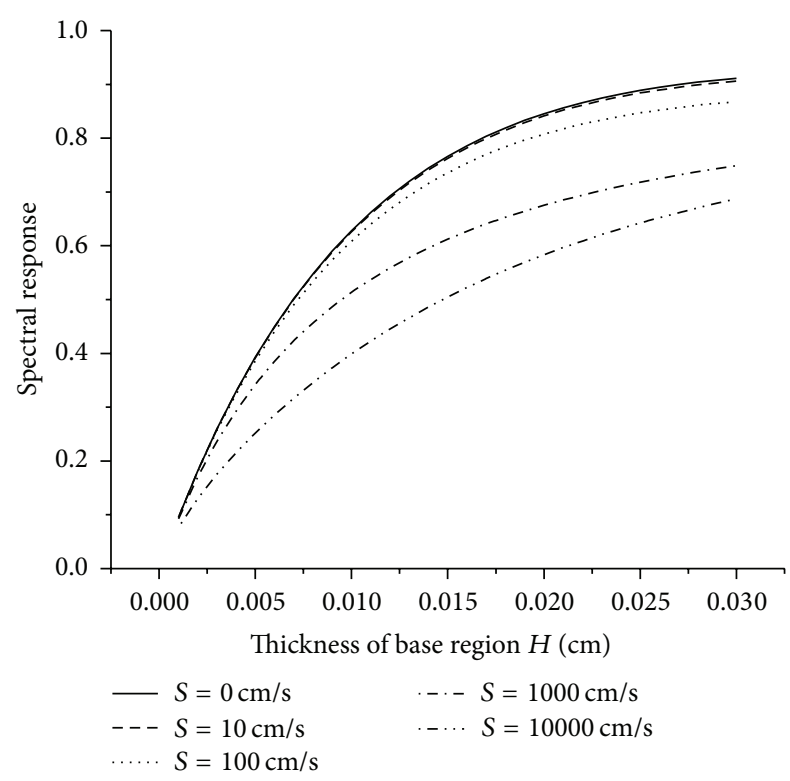

FIGURE 3: Spectral response of the SBSC as a function of thickness of the base region for different values of back surface recombination velocity $S$.

the equation given by Fossum [8]. The values of doping dependent carrier mobilities were taken from the published literature [9].

Figure 2 shows the variation of excess hole concentration as a function of position in the solar cell for different values of back surface recombination velocities.

It is observed that there is a large concentration of holes near the back surface, for smaller values of $S$, as compared to the case when high back surface recombination of carriers exists. For these higher values of $S$, there is large recombination of holes near the back surface, leading to

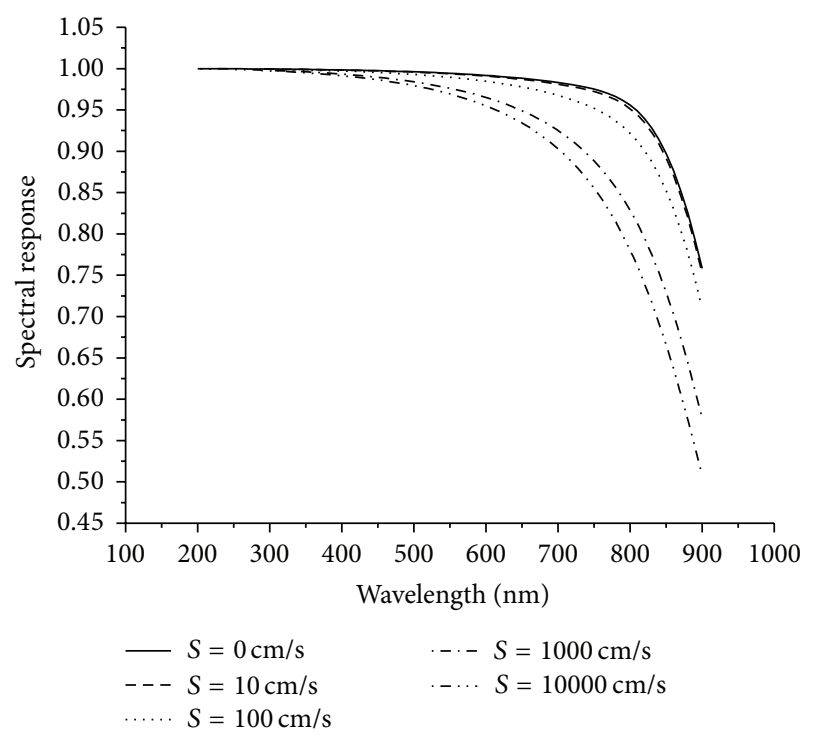

FIGURE 4: Spectral response of the SBSC as a function of wavelength for different values of back surface recombination velocity $S$.

smaller values of hole concentration there. Also, for these large values of $S$, there is steep gradient of minority carrier profile near the back surface, which gives rise to smaller values of photocurrent for these values of $S$.

In Figure 3, the variation of spectral response with base layer thickness is shown, for different values of back surface recombination velocity $S$. The results are consistent with the argument given in the discussion of Figure 2 that the photocurrent and hence spectral response in this case increase for smaller values of $S$. For larger thickness of base region, more light photons are absorbed and more photocurrent is obtained.

The plot of spectral response as a function of wavelength of incident light is shown in Figure 4, corresponding to different values of back surface recombination velocity $S$.

As expected from the results discussed in Figure 4, the spectral response increases significantly for smaller values of $S$. Also, the effect of $S$ on the spectral response is more visible for larger values of wavelength $\lambda$. This is because larger wavelengths are mostly absorbed near the back of the cell and the effect of back surface recombination on the photocurrent is pronounced for these larger wavelengths.

\section{Suggestion for a New Design of the SBSC for Improved Performance}

Results obtained show that the photocurrent of a SBSC is strongly dependent on the back surface recombination velocity $S$. The magnitude of photocurrent increases significantly for lower values of $S$.

It may be mentioned here that, in the development of conventional $\mathrm{n}^{+} \mathrm{p}$ junction solar cells, it was observed that incorporation of a highly doped $\mathrm{p}^{+}$layer at the back of the cell gave an $\mathrm{n}^{+} \mathrm{pp}^{+}$structure, which had much more improved photocurrent and open circuit voltage than that of the conventional structure [10]. The low-high $\left(\mathrm{pp}^{+}\right)$junction 


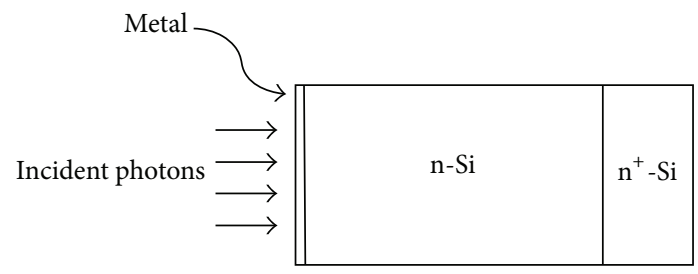

Figure 5: The proposed Schottky-barrier solar cell with a back surface field.

at the back of the cell gave rise to a back surface field, which effectively reduced the back surface recombination velocity of such cells [11]. These cells were called back surface field (BSF) solar cells.

Since it is observed here that the photocurrent of the SBSC increases significantly for lower values of $S$, taking a clue from the BSF $\mathrm{n}^{+} \mathrm{pp}^{+}$solar cells, we now suggest a new design of the SBSC with a back surface field, as shown in Figure 5. The width of the $\mathrm{p}^{+}$layer may be about $5 \mu \mathrm{m}$, and doping concentration may be kept at $10^{17} \mathrm{~cm}^{-3}$.

It is expected that this proposed new structure would give much improved photocurrent over the conventional SBSC. This is already evident from Figures 3 and 4, which shows much improved spectral response for smaller values of back surface recombination velocity.

\section{Conclusion}

The effect of back surface recombination velocity $S$ on the minority carrier distribution and photocurrent of a SBSC have been studied. It is observed that smaller values of $S$ give significantly higher values of photocurrent. Based on these results, a new design of a SBSC structure with a back surface field has been suggested, which is expected to give better performance, particularly with respect to the photocurrent of the cell.

\section{Conflict of Interests}

The authors declare that there is no conflict of interests regarding the publication of this paper.

\section{Acknowledgments}

The authors are grateful to the Department of Science and Technology, Government of India, for financial support under the DST-PURSE programme, granted to the University of Kalyani. They thank the authorities of the Indian Association for the Cultivation of Science, Kolkata, India, for allowing them to consult their library.

\section{References}

[1] D. M. Chapin, C. S. Fuller, and G. L. Pearson, "A new silicon p-n junction photocell for converting solar radiation into electrical power," Journal of Applied Physics, vol. 25, no. 5, pp. 676-677, 1954.
[2] A. W. Blakers and M. A. Green, "20\% Efficiency silicon solar cells," Applied Physics Letters, vol. 48, no. 3, pp. 215-217, 1986.

[3] A. Wang, J. Zhao, and M. A. Green, "24\% Efficient silicon solar cells," Applied Physics Letters, vol. 57, no. 6, pp. 602-604, 1990.

[4] D. L. Pulfrey and R. F. McOuat, "Schottky-barrier solar-cell calculations," Applied Physics Letters, vol. 24, no. 4, pp. 167-169, 1974.

[5] R. F. McOuat and D. L. Pulfrey, "A model for Schottky-barrier solar cell analysis," Journal of Applied Physics, vol. 47, no. 5, pp. 2113-2119, 1976.

[6] A. Chatterjee, A. K. Biswas, and A. Sinha, "Analytical study of the minority carrier distribution and photocurrent of a schottky-barrier silicon solar cell," International Journal of Renewable Energy Research, vol. 4, no. 2, pp. 504-507, 2014.

[7] H. J. Hovel, Semiconductors and Semimetals, vol. 11 of Solar Cells, Academic Press, New York, NY, USA, 1975.

[8] J. G. Fossum, "Computer-aided numerical analysis of silicon solar cells," Solid State Electronics, vol. 19, no. 4, pp. 269-277, 1976.

[9] D. M. Caughey and R. E. Thomas, "Carrier mobilities in silicon empirically related to doping and field," Proceedings of the IEEE, vol. 55, no. 12, pp. 2192-2193, 1967.

[10] J. Mandelkorn and J. H. Lamneck Jr., "A new electric field effect in silicon solar cells," Journal of Applied Physics, vol. 44, no. 10, pp. $4785-4787,1973$.

[11] A. Sinha and S. K. Chattopadhyaya, "Effect of heavy doping on the properties of high-low junction," IEEE Transactions on Electron Devices, vol. 25, no. 12, pp. 1412-1414, 1978. 


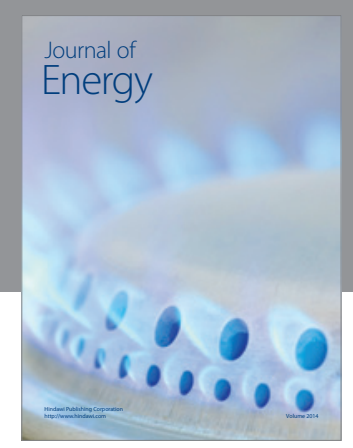

Journal of

Industrial Engineering
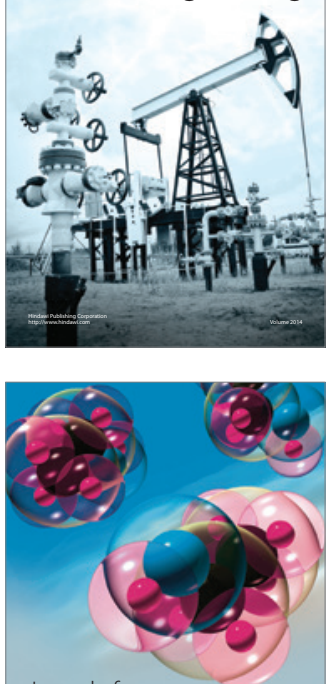

Fuels
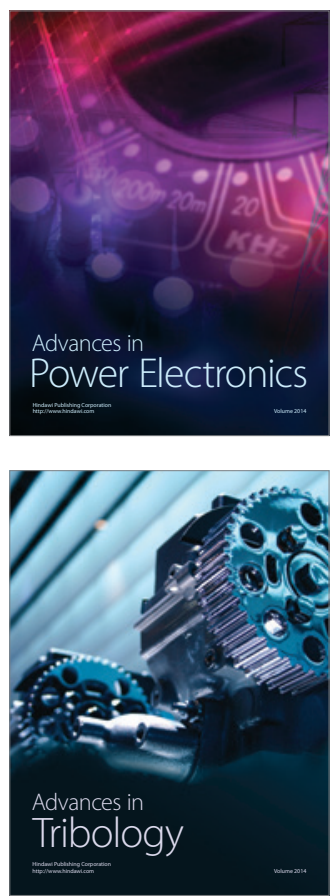

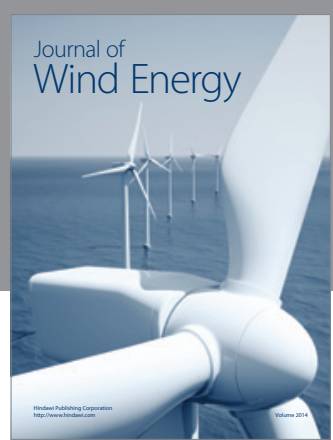

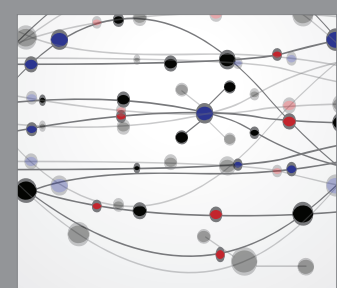

The Scientific World Journal

Submit your manuscripts at http://www.hindawi.com

Journal of

Structures
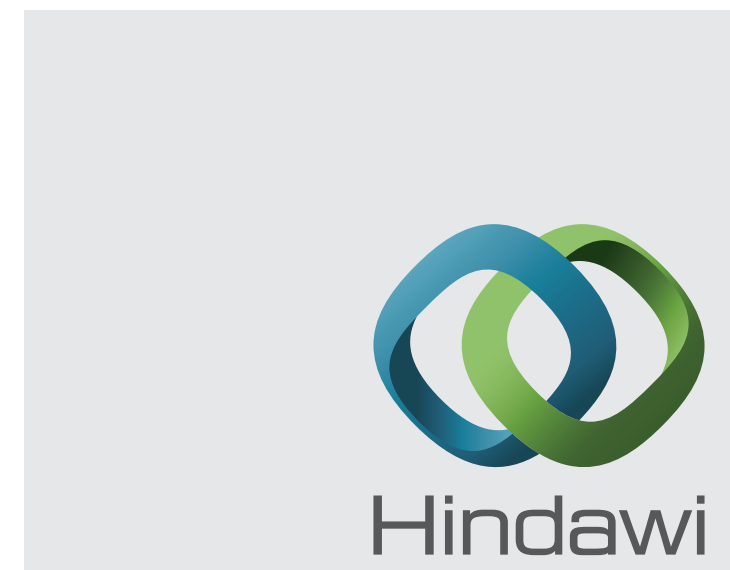

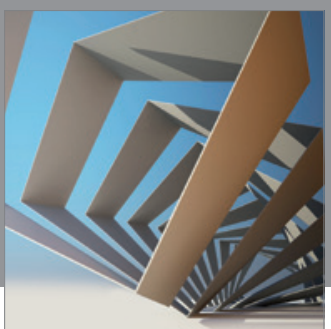

Rotating

Machinery
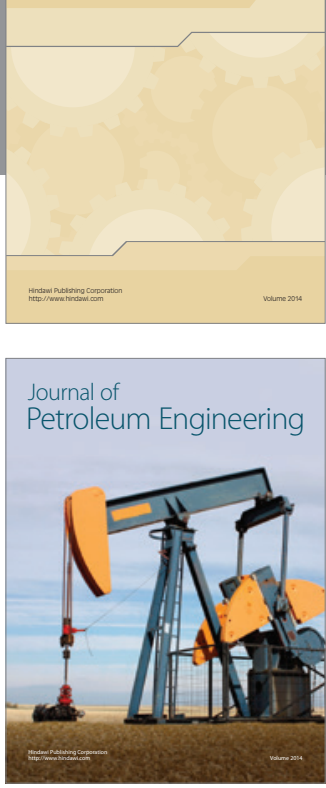

Journal of

Solar Energy
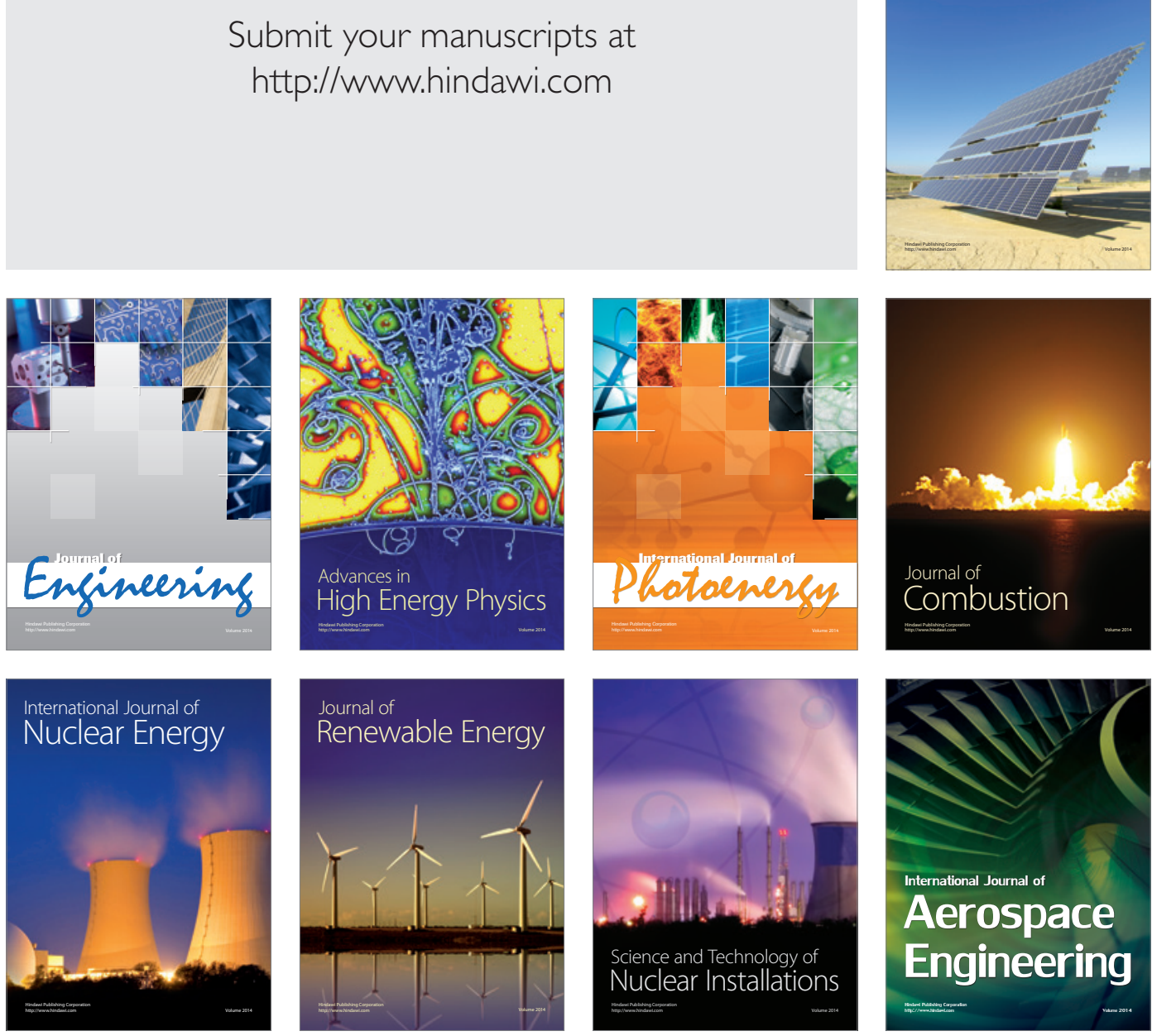